\title{
Semiclassical Strings, Dipole Deformations
}

\section{of $\mathcal{N}=1 \mathrm{SYM}$ and Decoupling of KK Modes}

\author{
N.P. Bobev ${ }^{\star \dagger}$, H. Dimov ${ }^{\ddagger}$ and R.C. Rashkov ${ }^{\dagger 1}$ \\ * Department of Physics and Astronomy, University of Southern \\ California, Los Angeles, CA 90089-0484, USA \\ ¥Department of Mathematics, University of Chemical Technology and \\ Metallurgy, 1756 Sofia, Bulgaria \\ $\dagger^{\dagger}$ Department of Physics, Sofia University, 1164 Sofia, Bulgaria
}

\begin{abstract}
In this paper we investigate the recently found $\gamma$-deformed MaldacenaNunez background by studying the behavior of different semiclassical string configurations. This background is conjectured to be dual to dipole deformations of $\mathcal{N}=1 \mathrm{SYM}$. We compare our results to those in the pure Maldacena-Nunez background and show that the energies of our string configurations are higher than in the undeformed background. Thinking in the lines of (hep-th/0505100) we argue that this is an evidence for better decoupling of the Kaluza-Klein modes from the pure SYM theory excitations. Moreover we are able to find a limit of the background in which the string energy is independent of $\gamma$, these strings are interpreted as corresponding to pure gauge theory effects.
\end{abstract}

\footnotetext{
${ }^{1}$ e-mail: rash@phys.uni-sofia.bg; bobev@usc.edu
} 


\section{Introduction}

In the recent years the AdS/CFT correspondence [1] became the major analytical tool for studying gauge theories at strong coupling. Thus it is of great interest to extend it to less supersymmetric theories which are phenomenologically more appropriate.

Recently Lunin and Maldacena [4] found the supergravity dual of the $\beta$-deformed $\mathcal{N}=4 \mathrm{SYM}[5]$ by performing an $S L(3, R)$ transformation on the well known $A d S_{5} \times S^{5}$ background. This provides new possibilities for quantitative checks of the AdS/CFT correspondence. When the deformation parameter $\beta$ is real (these are called $\gamma$ deformations) we have a continuous parameter which controls the deformation. So we can extend our knowledge for the correspondence between $A d S_{5} \times S^{5}$ and $\mathcal{N}=4$ SYM to this deformed case. This idea was first explored in [12] where the string energies in the $s u(2)$ sector where matched to the anomalous dimension of the corresponding operator in the $\gamma$-deformed $\mathcal{N}=4$ SYM (This technique was applied to $A d S_{5} \times S^{5}$ [21]-[39], similar considerations for other less supersymmetric backgrounds can be found in [40]-[55]). In [11] the integrability of the bosonic string on the Lunin-Maldacena background was proven and a new deformed background with three real deformations parameters $\gamma_{i}$ was found. The $s u(3)$ sector of semiclassical strings in this more general three parameter background was studied in [13] and a remarkable match between string energies and anomalous dimensions was shown. Different semiclassical rotating strings with spins in both the $A d S_{5}$ and the $\gamma$-deformed $S^{5}$ were studied in [14]. More interesting results in the study of this deformed theories can be found in [15]-[19].

Following the ideas in [4] Gursoy and Nunez [6] found a new interesting background which is a $\beta$-deformation of the well known Maldacena-Nunez background [7]. The Maldacena-Nunez background is dual to $N=1 \mathrm{SYM}$ and the authors of [6] provided evidence that the new deformed background is dual to the so called "dipole deformation" of the field theory. Moreover they were able to show that in the case of real deformation the pure gauge dynamics does not depend on the deformation parameter and the KaluzaKlein $(\mathrm{KK})$ modes increase their masses (respectively their energy). This is a new idea which gives a possibility to approach the problem of the mixing between the KK modes and the pure gauge theory effects. Motivated by this 
idea $^{2}$. For $\beta$-deformations and KK modes see also [10], in the present paper we want to consider semiclassical strings in the deformed Maldacena-Nunez background and analyze the dependence of their energies on the deformation parameter $^{3}$. The idea is to analyze the role of the KK modes unavoidably present in string backgrounds with less supersymmetry. The key point is the conjecture suggested by Gursoy and Nunez that the gamma deformations affect only the contributions from KK modes and hence it can serve as a test for whether our results are purely gauge theory effects or not. The conjecture explores the following observations. Starting from particular string background, in order to use AdS/CFT correspondence one should consider a UV completion of the corresponding supergravity solution. The completion, when we consider $\mathrm{N}=1$ case, depends on the KK modes. Deforming the background we change the behavior of the string theory (i.e. we are producing a family of completions parameterized by a continuous parameter $\gamma$ ) which results in changes in the spectrum, the functional relations between the spins $J_{1}, J_{2}$ and the energy etc. To make definite conclusions about gauge theory we should ensure that the result is entirely due to gauge theory effects. The satement made in [6] is that the deformation affect only the dynamics of the KK modes. Therefore, from practical point of view, one can trust only the result which do not depend on the deformation parameter, i.e. one should make computations in the undeformed and deformed backgrounds and the results depending on $\gamma$ have contributions from the KK modes, but the others are purely gauge theory effects.

In this paper we consider the deformed Maldacena-Nunez background and study the impact of the deformations on the semiclassical rotating and pulsating strings. Semiclassical strings in the Maldacena-Nunez background were studied in [20]. There the cases of rotating and pulsating strings were considered and the energy of the strings were expressed in terms of their angular momenta and quantum numbers respectively. In sections 3 and 4 of the present paper we consider the same ansatz as in [20] both for rotating and pulsating strings. We find the energies of the rotating strings in terms of their angular momenta in the limit of long and short strings. For the pulsating string ansatz we use the Bohr-Somerfeld quantization to compute the energies of the strings in terms of their quantum number $n$ and the

\footnotetext{
${ }^{2}$ We thank Carlos Nunez for suggesting this problem to us

${ }^{3}$ For extended discussion about deformations of flows from type IIB supergravity see also [8].
} 
winding number $m$. In both cases we reproduce the results of Pons and Talavera in the limit $\gamma \rightarrow 0$, this should be expected since this is the limit in which the background is not deformed (i.e. the original Maldacena-Nunez background is reproduced). More interestingly in both cases we find that the string energies increase due to the deformatioan. This can be interpreted as an evidence for the prediction of Gursoy and Nunez [6] for better decoupling of the KK modes in the deformed theory. In [6] it was conjectured that the sectors in which the deformation is decoupled should correspond to pure gauge theory effects. Fortunately, we were able to find a particular string ansatz in which there is non-trivial decoupling of the gamma deformation from the rest. In the concluding section we give detailed comments on the results of our study.

\section{$2 \tilde{\gamma}$ transformation of the Maldacena-Nunez background}

Here we will briefly review the background obtained by applying the LuninMaldacena procedure [4] to the so called Maldacena-Nunez background [7]. This $\gamma$ deformed background was found in [6] and it was argued that it is dual to dipole deformations of $N=1 \mathrm{SYM}$ theory. Moreover the authors of [6] conjectured that in this deformed background the KK modes decouple better from the pure gauge theory excitations and this is controlled by the real deformation parameter ${ }^{4}$. Following the notation of [6] we can write the metric of the Maldacena-Nunez background in the following form (this is different from the form in which the original solution was written but it is more convenient for applying the $S L(3, R)$ transformation):

$$
\begin{aligned}
& d s_{\text {string }}^{2}=e^{\phi}\left[d x_{1,3}^{2}+\alpha^{\prime} g_{s} N d r^{2}\right]+D_{1} d \psi^{2}+D_{2} d \theta^{2}+D_{3} d \tilde{\theta}+E_{1} d \theta d \tilde{\theta}+ \\
& E_{2} d \theta d \psi+E_{3} d \tilde{\theta} d \psi+\frac{F}{\sqrt{\Delta}}\left[d \varphi+\left(\alpha_{1}-C \beta_{1}\right) d \theta+\left(\alpha_{2}-C \beta_{2}\right) d \tilde{\theta}+\left(\alpha_{3}-C \beta_{3}\right) d \psi-C d \tilde{\varphi}\right]^{2}+ \\
& F \sqrt{\Delta}\left[d \tilde{\varphi}+\beta_{1} d \theta+\beta_{2} d \tilde{\theta}+\beta_{3} d \psi\right]^{2}
\end{aligned}
$$

\footnotetext{
${ }^{4}$ See [6] for an extensive discussion of this argument and some evidences supporting it
} 
If we define

$$
f=4 e^{2 h} \sin ^{2} \theta+\cos ^{2} \theta+a^{2} \sin ^{2} \theta \quad g=a \sin \theta \sin \tilde{\theta} \cos \psi-\cos \theta \cos \tilde{\theta}(2.2)
$$

We can write the functions appearing in (2.1) as

$$
\begin{aligned}
& F=\frac{\alpha^{\prime} g_{s} N e^{\phi}}{4} \sqrt{f-g^{2}} ; \quad \Delta=\frac{f-g^{2}}{f^{2}} ; \quad C=\frac{g}{f} ; \quad a(r)=\frac{2 r}{\sinh 2 r} ; \\
& \beta_{1}=\frac{f}{f-g^{2}} a \sin \psi \sin \tilde{\theta} ; \quad \beta_{2}=\frac{g}{f-g^{2}} a \sin \psi \sin \theta ; \quad \beta_{3}=\frac{f \cos \tilde{\theta}+g \cos \theta}{f-g^{2}} ; \\
& \alpha_{1}=\frac{a g \sin \tilde{\theta} \sin \psi}{f-g^{2}} ; \quad \alpha_{2}=\frac{a \sin \theta \sin \psi}{f-g^{2}} ; \quad \alpha_{3}=\frac{\cos \theta+g \cos \tilde{\theta}}{f-g^{2}} ; \\
& D_{1}=\frac{\alpha^{\prime} g_{s} N e^{\phi}}{4\left(f-g^{2}\right)}\left[f \sin ^{2} \tilde{\theta}-g^{2}-\cos ^{2} \theta-2 g \cos \theta \cos \tilde{\theta}\right] ; \\
& D_{2}=\frac{\alpha^{\prime} g_{s} N e^{\phi}}{4}\left[a^{2}+4 e^{2 h}-\frac{f}{f-g^{2}} a^{2} \sin { }^{2} \psi \sin { }^{2} \tilde{\theta}\right] ; \quad D_{3}=\frac{\alpha^{\prime} g_{s} N e^{\phi}}{4}\left[1-\frac{a^{2} \sin ^{2} \theta \sin ^{2} \psi}{f-g^{2}}\right] ; \\
& E_{1}=\frac{a \alpha^{\prime} g_{s} N e^{\phi}}{2}\left[\cos \psi-\frac{g}{f-g^{2}} a \sin ^{2} \psi \sin \theta \sin \tilde{\theta}\right] ; \\
& E_{2}=-\frac{a \alpha^{\prime} g_{s} N e^{\phi}}{2} \frac{\sin \psi \sin \tilde{\theta}(f \cos \tilde{\theta}+g \cos \theta)}{f-g^{2}} ; \\
& E_{3}=-\frac{a \alpha^{\prime} g_{s} N e^{\phi}}{2} \frac{\sin \psi \sin \theta(\cos \theta+g \cos \tilde{\theta})}{f-g^{2}}
\end{aligned}
$$

There are other fields present in the background but we are not going to present their explicit form. After performing an $S L(3, R)$ transformation 
(which is equivalent to a TsT transformation [11]) the metric takes the form:

$$
\begin{aligned}
& \left(d s_{\text {string }}^{2}\right)^{\prime}=\left(\frac{e^{2\left(\Phi^{\prime}-\Phi\right)} F}{F^{\prime}}\right)^{\frac{1}{3}}\left[e^{\phi}\left[d x_{1,3}^{2}+\alpha^{\prime} g_{s} N d r^{2}\right]+D_{1} d \psi^{2}+D_{2} d \theta^{2}+D_{3} d \tilde{\theta}+E_{1} d \theta d \tilde{\theta}+\right. \\
& \left.E_{2} d \theta d \psi+E_{3} d \tilde{\theta} d \psi\right]+\frac{F^{\prime}}{\sqrt{\Delta}}\left[d \varphi+\left(\alpha_{1}-C \beta_{1}\right) d \theta+\left(\alpha_{2}-C \beta_{2}\right) d \tilde{\theta}+\left(\alpha_{3}-C \beta_{3}\right) d \psi-C d \tilde{\varphi}\right]^{2}+ \\
& F^{\prime} \sqrt{\Delta}\left[d \tilde{\varphi}+\beta_{1} d \theta+\beta_{2} d \tilde{\theta}+\beta_{3} d \psi\right]^{2}
\end{aligned}
$$

where $\Phi$ and $\Phi^{\prime}$ are the original and the transformed dilaton field, $F^{\prime}=$ $\frac{F}{1+\tilde{\gamma}^{2} F^{2}}$ and $\tilde{\gamma}$ is the real transformation parameter. We would like to note that it can be proven that this new deformed geometry does not generates any new singularities with respect to the undeformed one. After this brief comments on the deformed Maldacena-Nunez background we proceed with the investigations of the behavior of various classical string configurations in this geometry.

\section{$3 \quad$ Rotating strings in $S^{2} \times \mathbb{R}$}

In the following sections we are going to study different string configurations in the above presented $\tilde{\gamma}$-deformed background. We will focus our attention on the dependence of the string energy on the real deformation parameter $\gamma$ since we want to test the proposal made in [6] for better decoupling of the KK modes. Since we want to compare our results to the case of the pure Maldacena-Nunez background, we will work with the same string configurations as those studied by Pons and Talavera [20]. We start with the following simple rotating string ansatz:

$$
t=\kappa \tau \quad \varphi=\kappa \omega \tau \quad \theta=\frac{\pi}{2} \quad r=r(\sigma)
$$

The relevant part of the deformed metric is:

$$
d s^{2}=\left(\frac{e^{2\left(\Phi^{\prime}-\Phi\right)} F}{F^{\prime}}\right)^{\frac{1}{3}}\left(-e^{\Phi} d t^{2}+e^{\Phi} A d r^{2}\right)+\frac{F^{\prime}}{\sqrt{\Delta}} d \varphi^{2}
$$


where

$$
\begin{gathered}
A=\alpha^{\prime} g_{s} N \quad F=\frac{A e^{\Phi}}{4} \sqrt{4 e^{2 h}+a^{2}(r)} \quad F^{\prime}=\frac{F}{1+\tilde{\gamma}^{2} F^{2}} \\
e^{2 \Phi^{\prime}}=\frac{e^{2 \Phi}}{1+\tilde{\gamma}^{2} F^{2}} \quad \Delta=\frac{1}{4 e^{2 h}+a^{2}(r)} \quad a(r)=\frac{2 r}{\sinh 2 r} \\
e^{2 h}=r \operatorname{coth} 2 r-\frac{r^{2}}{\sinh ^{2} 2 r}-\frac{1}{4} \quad e^{2 \Phi}=e^{2 \Phi_{0}} \frac{\sinh 2 r}{2 e^{h}}
\end{gathered}
$$

For later convenience we define

$$
\beta(r)=e^{2 h(r)}+\frac{a^{2}(r)}{4}
$$

Using these definitions we can rewrite the components of the metric as follows

$$
\begin{gathered}
G_{t t}=-e^{\Phi} \quad G_{r r}=A e^{\Phi} \\
G_{\varphi \varphi}=\frac{A e^{\Phi} \beta(r)}{1+\frac{\tilde{\gamma}^{2}}{4} A^{2} e^{2 \Phi} \beta(r)}
\end{gathered}
$$

The Polyakov action for this ansatz is simply

$$
S=-\frac{1}{4 \pi} \int d \tau d \sigma\left(e^{\Phi} \kappa^{2}+A e^{\Phi}\left(r^{\prime}(\sigma)\right)^{2}-\frac{A e^{\Phi} \beta(r) \kappa^{2} \omega^{2}}{1+\frac{\tilde{\gamma}^{2}}{4} A^{2} e^{2 \Phi} \beta(r)}\right)
$$

We should also impose the Virasoro constraints in order to ensure conformal invariance. One of them is trivially satisfied by our ansatz and from the second one we obtain the following equation for $r(\sigma)$ :

$$
A\left(r^{\prime}(\sigma)\right)^{2}=\kappa^{2}\left(1-\frac{A \beta(r) \omega^{2}}{1+\frac{\tilde{\gamma}^{2}}{4} A^{2} e^{2 \Phi} \beta(r)}\right)
$$

In the limit $\tilde{\gamma} \rightarrow 0$ we recover the equation for the pure Maldacena-Nunez case, as should be expected. This equation can be rewritten as: 


$$
d \sigma=d r \frac{\sqrt{A}}{\kappa} \frac{1}{\sqrt{1-\frac{A \beta(r) \omega^{2}}{1+\frac{\tilde{\gamma}^{2}}{4} A^{2} e^{2 \Phi} \beta(r)}}}
$$

This relation is useful for the computation of the conserved charges in our problem (the energy and the angular momentum) which adopt the form:

$$
\begin{gathered}
E=\frac{\kappa}{2 \pi} \int d \sigma e^{\Phi} \\
J=\frac{A \kappa \omega}{2 \pi} \int d \sigma \frac{e^{\Phi} \beta(r)}{1+\frac{\tilde{\gamma}^{2}}{4} A^{2} e^{2 \Phi} \beta(r)}
\end{gathered}
$$

In order to compute these quantities we need the explicit form of the dilaton field and the function $\beta(r)$

$$
\begin{gathered}
e^{2 \Phi}=\frac{e^{2 \Phi_{0}} \sinh ^{2} 2 r}{\sqrt{4 r \cosh 2 r \sinh 2 r-\sinh ^{2} 2 r-4 r^{2}}} \\
\beta(r)=r \operatorname{coth} 2 r-\frac{1}{4}
\end{gathered}
$$

where $\Phi_{0}$ is the value of the dilaton field at the point $r=0$. Substituting these function into the expressions for the energy and the angular momentum leads to analytically non-solvable integrals and we should make some approximations. Since we are interested in the way which the deformation parameter affects the energy we will consider the limits of long and short strings and try to extract the leading terms for the energy and angular momentum.

\subsection{Long strings}

The limit $r_{0} \rightarrow \infty$, where $r_{0}$ is the turning point is called long strings. In this limit the dilaton and the function $\beta(r)$ have the following behavior:

$$
\beta(r) \approx r \quad e^{\Phi(r)} \approx \frac{e^{r}}{2 r^{\frac{1}{4}}}
$$


So in this approximation the conserved charges are

$$
\begin{gathered}
E=\frac{\sqrt{A}}{2 \pi} \int_{0}^{r_{0}} d r \frac{e^{r}}{2 r^{\frac{1}{4}}} \frac{1}{\sqrt{1-\frac{16 \omega^{2} A r}{16+\tilde{\gamma}^{2} A^{2} e^{2 r} \sqrt{r}}}} \\
J=\frac{A^{\frac{3}{2}} \omega}{2 \pi} \int_{0}^{r_{0}} d r \frac{e^{r} r^{\frac{3}{4}}}{2\left(1+\frac{\tilde{\gamma}^{2}}{16} A^{2} e^{2 r} \sqrt{r}\right)} \frac{1}{\sqrt{1-\frac{16 \omega^{2} A r}{16+\tilde{\gamma}^{2} A^{2} e^{2 r} \sqrt{r}}}}
\end{gathered}
$$

Using the long string approximation we can solve this integrals, after some calculations we end up with the following expressions:

$$
\begin{aligned}
& E \approx \frac{\sqrt{A}}{4 \sqrt{\pi}} \frac{\Gamma(3 / 4)}{\Gamma(5 / 4)} r_{0}^{3 / 4}{ }_{1} F_{1}\left[\frac{3}{4}, \frac{5}{4}, r_{0}\right] \stackrel{r_{0} \gg}{\rightarrow} \frac{\sqrt{A}}{4 \sqrt{\pi}} r_{0}^{1 / 4} e^{r_{0}} \\
& \omega J \approx \frac{\sqrt{A}}{4 \sqrt{\pi}} r_{0}^{3 / 4} \frac{\Gamma(7 / 4)}{\Gamma(9 / 4)}{ }_{1} F_{1}\left[\frac{7}{4}, \frac{9}{4}, r_{0}\right] \stackrel{r_{0} \gg}{\rightarrow} \frac{\sqrt{A}}{4 \sqrt{\pi}} r_{0}^{1 / 4} e^{r_{0}} \\
& E-\omega J \approx \frac{\sqrt{A}}{8 \sqrt{\pi}} r_{0}^{3 / 4} \frac{\Gamma(3 / 4)}{\Gamma(9 / 4)}{ }_{1} F_{1}\left[\frac{3}{4}, \frac{9}{4}, r_{0}\right] \stackrel{r_{0} \gg}{\rightarrow} \frac{\sqrt{A}}{8 \sqrt{\pi}} r_{0}^{-3 / 4} e^{r_{0}}
\end{aligned}
$$

Our results look exactly the same as those of Pons and Talavera but in our case the turning point is:

$$
r_{0}=\frac{16+\tilde{\gamma}^{2} A^{2} e^{2 r_{0}} \sqrt{r_{0}}}{\omega^{2} A}
$$

So the effect of the deformation parameter essentially increases the energy and the angular momentum. Of course in the limit $\tilde{\gamma} \rightarrow 0$ we reproduce exactly the results from the undeformed case.

We can easily calculate the ratio:

$$
\frac{E-\omega J}{\omega J} \approx \frac{1}{2 r_{0}}
$$

So as in the undeformed case this is a finite value. It aslo useful to extract the dependance of the string energy on the angular momentum. The leading term in this dependance (we assume large values of $E$ and $J$ ) is: 


$$
E \approx \frac{1}{R} \frac{J}{\ln ^{1 / 2}\left(\frac{4 \sqrt{\pi}}{R} \omega J\right)} \sqrt{16+\tilde{\gamma}^{2} R^{2} 16 \pi \omega^{2} J^{2} \ln ^{1 / 2}\left(\frac{4 \sqrt{\pi}}{R} \omega J\right)}
$$

One can check that this messy expression reduces to the leading term for the energy in the work of Pons and Talavera.

\subsection{Short strings}

Here we will present a similar analysis to the previous section but in the limit of short strings (i.e. $r_{0} \rightarrow 0$ ). In this limit the dilaton field and the function $\beta(r)$ have the following behavior:

$$
\beta(r) \approx \frac{1}{4}+\frac{2}{3} r^{2} \quad e^{\Phi(r)} \approx 1+\frac{4}{9} r^{2}
$$

Substituting these approximate values into the expressions for the energy and the angular momentum leads to:

$$
\begin{aligned}
& E=\frac{\sqrt{A}}{2 \pi \sqrt{\xi}} \int_{0}^{r_{0}} d r \frac{\left(1+\frac{4}{9} r^{2}\right)}{\sqrt{r_{0}^{2}-r^{2}}} \\
& J=\frac{A^{\frac{3}{2}} \omega}{2 \pi \sqrt{\xi}} \int_{0}^{r_{0}} d r \frac{\left(\frac{1}{4}+\frac{7}{9} r^{2}\right)}{\sqrt{r_{0}^{2}-r^{2}}}
\end{aligned}
$$

where $r_{0}$ is the turning point and $\xi$ is a short notation for the following expression:

$$
\begin{gathered}
\xi=\frac{32 A\left(3 \omega^{2}-\tilde{\gamma}^{2} A\right)}{9\left(16+\tilde{\gamma}^{2} A^{2}\right)} \\
r_{0}^{2}=\frac{9\left(16+\tilde{\gamma}^{2} A^{2}-4 \omega^{2} A\right)}{32 A\left(3 \omega^{2}-\tilde{\gamma}^{2} A\right)}
\end{gathered}
$$


After some straightforward but tedious calculations we end up with the following expressions for the energy and angular momentum:

$$
\begin{gathered}
E \approx \sqrt{\frac{3}{2}} \frac{1}{\omega} \sqrt{\frac{1+\frac{\tilde{\gamma}^{2} A^{2}}{16}}{1-\frac{\tilde{\gamma}^{2} A}{3 \omega^{2}}}} \\
J \approx \sqrt{\frac{3}{2} \frac{1}{\omega^{2}}}\left(\frac{1+\frac{\tilde{\gamma}^{2} A^{2}}{16}}{1-\frac{\tilde{\gamma}^{2} A}{3 \omega^{2}}}\right)^{\frac{3}{2}}
\end{gathered}
$$

so that we can extract the following relation

$$
E^{2}=\sqrt{\frac{3}{2}}\left(\frac{1+\frac{\tilde{\gamma}^{2} A^{2}}{16}}{1-\frac{\tilde{\gamma}^{2} A}{3 \omega^{2}}}\right)^{-\frac{1}{2}} J
$$

This is an expected Regge type behavior for the energy of a very short string, as before if we take the limit $\tilde{\gamma} \rightarrow 0$ we reproduce the analogous relation found by Pons and Talavera. Looking at the expression for the energy (3.20) we see that the deformation parameter again increases the energy and the angular momentum. Thus we can conclude that for this rotating string configuration the energies are shifted in the positive direction, which comes as an evidence for the conjecture for better decoupling of the KK modes in the $\gamma$-deformed geometry. Encouraged by this observation and looking for similar pattern we proceed with the study of semiclassical pulsating strings.

\section{Pulsating strings in $S^{2} \times \mathbb{R}$}

Pulsating strings represent another interesting class of semiclassical strings used as a tool for studying the $A d S / C F T$ correspondence [25]-[30]. We use the approach of Minahan [27] and the following pulsating string ansatz:

$$
t=\tau \quad \varphi=m \sigma \quad \theta=\frac{\pi}{2} \quad r=r(\tau)
$$


The Nambu-Goto Lagrangian for this ansatz is

$$
\mathcal{L}_{N G}=m e^{\Phi} \sqrt{\frac{A \beta(r)\left(1-A \dot{r}^{2}\right)}{1+\frac{\tilde{\gamma}^{2}}{4} A^{2} e^{2 \Phi} \beta(r)}}
$$

The canonical momentum corresponding to $r$ can be obtained from this Lagrangian:

$$
\Pi_{r}=\frac{1}{1+\frac{\tilde{\gamma}^{2}}{4} A^{2} e^{2 \Phi} \beta(r)} \frac{m e^{\Phi} A^{2} \beta(r) \dot{r}}{\sqrt{\frac{A \beta(r)\left(1-A \dot{r}^{2}\right)}{1+\frac{\tilde{\gamma}^{2}}{4} A^{2} e^{2 \Phi} \beta(r)}}}
$$

Now we can easily compute the Hamiltonian of our system

$$
H=\sqrt{\frac{\Pi_{r}^{2}}{A}+\frac{m^{2} A e^{2 \Phi} \beta(r)}{1+\frac{\tilde{\gamma}^{2}}{4} A^{2} e^{2 \Phi} \beta(r)}}
$$

This is the Hamiltonian of a one dimensional system with the potential:

$$
V=\frac{m^{2} A^{2} e^{2 \Phi} \beta(r)}{1+\frac{\tilde{\gamma}^{2}}{4} A^{2} e^{2 \Phi} \beta(r)}
$$

In order to compute the energy levels we will make use of the WKB approximation, namely

$$
I=\int_{0}^{r_{0}} d r \sqrt{A E^{2}-\frac{m^{2} A^{2} e^{2 \Phi} \beta(r)}{1+\frac{\tilde{\gamma}^{2}}{4} A^{2} e^{2 \Phi} \beta(r)}}=\left(n+\frac{1}{2}\right) \pi
$$

Unfortunately this integral is analytically unsolvable and we should again make use of some appropriate approximations. As in the previous section we will focus on the limits of short and long strings and find the leading contributions to the energy. 


\subsection{Long strings}

The limit of long strings $r_{0} \rightarrow \infty$ is physically interesting and it greatly simplifies the form of the potential, which has the following asymptotic behavior:

$$
V=\frac{\frac{m^{2} A^{2}}{4} e^{2 r} \sqrt{r}}{1+\frac{\tilde{\gamma}^{2} A^{2}}{16} e^{2 r} \sqrt{r}}
$$

Now we can solve the equation $A E^{2}=V\left(r_{0}\right)$ for the turning point $r_{0}$, of course we again explore the approximation $r_{0} \rightarrow \infty$. After some computations we find:

$$
r_{0} \approx \frac{1}{2} \ln \left(\frac{4 E^{2}}{m^{2} A-\frac{\tilde{\gamma}^{2} A^{2} E^{2}}{4}}\right)
$$

The integral $I$ in this limit has the following leading behavior:

$$
I \approx \sqrt{A} E r_{0}+\mathcal{O}\left(\frac{1}{E}\right)
$$

So we can substitute our expression for $r_{0}$ and end up with:

$$
I \approx \frac{1}{2} \sqrt{A} E \ln \left(\frac{4 E^{2}}{m^{2} A-\frac{\tilde{\gamma}^{2} A^{2} E^{2}}{4}}\right)+\mathcal{O}\left(\frac{1}{E}\right)
$$

We can compare our result to the one from the paper of Pons and Talavera. In the limit $\tilde{\gamma} \rightarrow 0$ we reproduce their result as expected, because this is the limit which reproduces the original Maldacena-Nunez background. Moreover since $I=\left(n+\frac{1}{2}\right) \pi$ we see that the energy levels are again shifted upwards, which coincides with the pattern found in the previous section for rotating strings.

\subsection{Short strings}

We will consider the limit $r_{0} \rightarrow 0$ (i.e. short strings). In this limit the potential adopts the following simpler form:

$$
V(r)=m^{2} A^{2}\left(\frac{1}{1+\frac{\tilde{\gamma}^{2} A^{2}}{4}}+r^{2}\right)
$$


Using this we can find the turning point $r_{0}$, i.e. the solution of the equation $E^{2}=V\left(r_{0}\right)$

$$
r_{0}=\frac{\sqrt{A\left(4+\tilde{\gamma}^{2} A^{2}\right)\left(4 E^{2}+E^{2} \tilde{\gamma}^{2} A^{2}-4 m^{2} A\right)}}{m\left(4 A+\tilde{\gamma}^{2} A^{3}\right)}
$$

Now the integral $I=\int_{0}^{r_{0}} d r \sqrt{E^{2}-V(r)}$ can be solved but the result is to long and we will not present it here. The important thing is that we can further solve the equation for $E^{2}$ in terms of the oscillator level $n$

$$
E^{2} \approx\left(\frac{\sqrt{A}+A^{\frac{5}{2}} \tilde{\gamma}^{2}}{1+\frac{\tilde{\gamma}^{2} A^{2}}{4}}\right) m n
$$

Amazingly in the limit $\tilde{\gamma} \rightarrow 0$ this expression reduces to the result of Pons and Talavera. However here it is not that obvious that the energy is increased but since in the undeformed case $E^{2}=\sqrt{A} m n$ we can say that (4.13) is affected by the deformation parameter and indeed it is greater than the energy of the string in the undeformed geometry.

\section{5 "Fast" rotating strings in $S^{3}$}

In this case, the discussion follows closely the one in the $\gamma$-deformed $S^{3}$ part. If we put

$$
\theta=0, r=0, \varphi=\text { const }
$$

the relevant part of the metric is

$d s^{2}=-e^{\Phi_{0}} d t^{2}+A d \tilde{\theta}^{2}+\frac{A}{1+\gamma^{2} A^{2} \sin ^{2} \tilde{\theta}}(d \psi+\cos \tilde{\theta} d \tilde{\varphi})^{2}+\frac{A \sin ^{2} \tilde{\theta}}{1+\gamma^{2} A^{2} \sin ^{2} \tilde{\theta}} d \tilde{\varphi}^{2}$,

where $A=\frac{\alpha^{\prime} g_{s} N e^{\Phi_{0}}}{4}$.

The B-field is $B_{2}=d \psi \wedge d \tilde{\varphi}$.

Let us now study the structure of the string sigma model action in the "2spin" sector in the limit of large total spin $J=J_{1}+J_{2}$. Setting

$$
\psi=\alpha+\xi, \quad \tilde{\varphi}=\alpha-\xi, \text { i.e. } \xi \equiv \frac{1}{2}(\psi-\tilde{\varphi})
$$


we may treat $\alpha$ as a "fast" angular variable while $\xi$ and $\tilde{\theta}$ will be "slow" variables whose time evolution will be suppressed. Using these definitions we can rewrite the metric as follows

$$
d s^{2}=-e^{\Phi_{0}} d t^{2}+A d \tilde{\theta}^{2}+\frac{4 A}{1+\gamma^{2} A^{2} \sin ^{2} \tilde{\theta}}\left[\cos ^{2}\left(\frac{\tilde{\theta}}{2}\right) d \alpha^{2}+\sin ^{2}\left(\frac{\tilde{\theta}}{2}\right) d \xi^{2}\right] .
$$

The B-field is $B_{2}=d \psi \wedge d \tilde{\varphi}=-2 d \alpha \wedge d \xi$, i. e. $B_{\alpha \xi}=-2$.

Then the relevant Polyakov action is

$$
\begin{aligned}
S & =-\frac{1}{4 \pi} \int d \tau d \sigma\left[\sqrt { - h } h ^ { p q } \left(-e^{\Phi_{0}} \partial_{p} t \partial_{q} t+A \partial_{p} \tilde{\theta} \partial_{q} \tilde{\theta}+\right.\right. \\
& \left.\left.+\frac{4 A \cos ^{2}\left(\frac{\tilde{\theta}}{2}\right)}{1+\gamma^{2} A^{2} \sin ^{2} \tilde{\theta}} \partial_{p} \alpha \partial_{q} \alpha+\frac{4 A \sin ^{2}\left(\frac{\tilde{\theta}}{2}\right)}{1+\gamma^{2} A^{2} \sin ^{2} \tilde{\theta}} \partial_{p} \xi \partial_{q} \xi\right)-2 \varepsilon^{p q} B_{\alpha \xi} \partial_{p} \alpha \partial_{q} \xi\right]
\end{aligned}
$$

To implement the uniform gauge fixing one may either consider the phasespace action and fix $p_{\alpha}=$ const or, equivalently, first do T-duality (i.e. 2-d duality) in $\alpha$ direction in the above action and gauge-fix $\tilde{\alpha}=J \sigma$. The components of the metric (5.3) after T-duality transformation take the form:

$$
\begin{gathered}
\tilde{G}_{\tilde{\alpha} \tilde{\alpha}}=\frac{1+\gamma^{2} A^{2} \sin ^{2} \tilde{\theta}}{4 A \cos ^{2}\left(\frac{\tilde{\theta}}{2}\right)} \equiv g_{1}, \quad \tilde{G}_{t t}=-e^{\Phi_{0}}, \quad \tilde{G}_{\tilde{\theta} \tilde{\theta}}=A, \\
\tilde{G}_{\xi \xi}=\frac{A^{2} \sin ^{2} \tilde{\theta}+\left(1+\gamma^{2} A^{2} \sin ^{2} \tilde{\theta}\right)^{2}}{A \cos ^{2}\left(\frac{\tilde{\theta}}{2}\right)\left(1+\gamma^{2} A^{2} \sin ^{2} \tilde{\theta}\right)} \equiv g_{2}, \tilde{G}_{\tilde{\alpha} \xi}=-2 \frac{\left(1+\gamma^{2} A^{2} \sin ^{2} \tilde{\theta}\right)}{4 A \cos ^{2}\left(\frac{\tilde{\theta}}{2}\right)}=-2 g_{1}
\end{gathered}
$$

and then we have

$$
d \tilde{s}^{2}=-e^{\Phi_{0}} d t^{2}+A d \tilde{\theta}^{2}+g_{1} d \tilde{\alpha}^{2}+g_{2} d \xi^{2}-4 g_{1} d \tilde{\alpha} d \xi .
$$

All the components of $\tilde{B}$-field are zero.

The action after T-duality takes the form:

$$
\begin{aligned}
S=-\frac{1}{4 \pi} \int d \tau d \sigma \sqrt{-h} h^{p q}\left[-e^{\Phi_{0}} \partial_{p} t \partial_{q} t\right. & +A \partial_{p} \tilde{\theta} \partial_{q} \tilde{\theta}+g_{1} \partial_{p} \tilde{\alpha} \partial_{q} \tilde{\alpha}+ \\
& \left.+g_{2} \partial_{p} \xi \partial_{q} \xi-2 g_{1} \partial_{p} \tilde{\alpha} \partial_{q} \xi\right]
\end{aligned}
$$


We are going to use Nambu-Goto action, because we don't know the gaugefixing of the $\sqrt{-h} h^{p q}$.

Imposing now the gauge ( here $\tau$ and $\sigma$ are world-sheet coordinates )

$$
t=\tau, \quad \tilde{\alpha}=J \sigma,
$$

and solving for the world-sheet metric, we find

$$
\begin{aligned}
-\operatorname{det} h_{p q}=[ & \left.A \dot{\tilde{\theta}} \tilde{\theta}^{\prime}+g_{2} \dot{\xi} \xi^{\prime}-2 g_{1} J \dot{\xi}\right]^{2} \\
& -\left[-e^{\Phi_{0}}+A \dot{\tilde{\theta}}+g_{2} \dot{\xi}^{2}\right]\left[A \tilde{\theta}^{\prime 2}+g_{1} J^{2}+g_{2}{\xi^{\prime}}^{2}-4 g_{1} J \xi^{\prime}\right] .
\end{aligned}
$$

In the simple case $\tilde{\theta}=$ const we have

$$
\sqrt{-\operatorname{det} h_{p q}}=\sqrt{e^{\Phi_{0}} g_{1} J^{2}-\left(g_{1} g_{2}-4 g_{1}^{2}\right) J^{2} \dot{\xi}^{2}+e^{\Phi_{0}} g_{2} \xi^{\prime 2}-4 e^{\Phi_{0}} g_{1} J \xi^{\prime}} .
$$

To isolate the sector of "fast strings" we should take J to be large, i.e. $\frac{1}{J^{2}} \rightarrow$ 0 , and $\dot{\xi}$ to be small ( $\xi$ is "slow" variable). Then

$$
\begin{aligned}
& \sqrt{-\operatorname{det} h_{p q}}=J \sqrt{e^{\Phi_{0}} g_{1}-\left(g_{1} g_{2}-4 g_{1}^{2}\right) \dot{\xi}^{2}-4 e^{\Phi_{0}} g_{1} \xi^{\prime} \frac{1}{J}+e^{\Phi_{0}} g_{2} \xi^{\prime^{2}} \frac{1}{J^{2}}}= \\
= & J\left[\sqrt{e^{\Phi_{0}} g_{1}-\left(g_{1} g_{2}-4 g_{1}^{2}\right) \dot{\xi}^{2}}-\frac{4 e^{\Phi_{0}} g_{1} \xi^{\prime}}{2 \sqrt{e^{\Phi_{0}} g_{1}-\left(g_{1} g_{2}-4 g_{1}^{2}\right) \dot{\xi}^{2}}}+O\left(\frac{1}{J}\right)\right] \approx \\
& \approx\left[J\left(e^{\Phi_{0}} g_{1}-\left(g_{1} g_{2}-4 g_{1}^{2}\right) \dot{\xi}^{2}\right)-2 e^{\Phi_{0}} g_{1} \xi^{\prime}\right] \frac{1}{\sqrt{e^{\Phi_{0}} g_{1}-\left(g_{1} g_{2}-4 g_{1}^{2}\right) \dot{\xi}^{2}}}= \\
= & {\left[J\left(e^{\Phi_{0}} g_{1}-\left(g_{1} g_{2}-4 g_{1}^{2}\right) \dot{\xi}^{2}\right)-2 e^{\Phi_{0}} g_{1} \xi^{\prime}\right]\left[\frac{1}{e^{\Phi_{0} / 2} \sqrt{g_{1}}}+\frac{\left(g_{1} g_{2}-4 g_{1}^{2}\right)}{2\left(e^{\Phi_{0}} g_{1}\right)^{3 / 2}} \dot{\xi}^{2}+o\left(\dot{\xi}^{4}\right)\right] } \\
& \approx-\frac{\sqrt{g_{1}}\left(g_{2}-4 g_{1}\right)}{2 e^{\Phi_{0} / 2}}\left(J+2 \xi^{\prime}\right) \dot{\xi}^{2}-2 e^{\Phi_{0} / 2} \sqrt{g_{1}} \xi^{\prime}+J e^{\Phi_{0} / 2} \sqrt{g_{1}} . \quad(5.11)
\end{aligned}
$$

Finally, the Nambu-Goto Lagrangian takes the form

$\mathcal{L}\left(\dot{\xi}, \xi^{\prime}\right)=-\frac{1}{2 \pi \alpha^{\prime}}\left[-\frac{\sqrt{g_{1}}\left(g_{2}-4 g_{1}\right)}{2 e^{\Phi_{0} / 2}}\left(J+2 \xi^{\prime}\right) \dot{\xi}^{2}-2 e^{\Phi_{0} / 2} \sqrt{g_{1}} \xi^{\prime}+J e^{\Phi_{0} / 2} \sqrt{g_{1}}\right]$. 
The ansatz we employ is

$$
\tilde{\theta}=\text { const }, \quad \xi \equiv \frac{1}{2}(\psi-\tilde{\varphi})=\omega \tau+\frac{1}{2} m \sigma,
$$

where $m=m_{1}-m_{2}$ is an integer and $\omega$ is a constant ( one can check that these are solutions of the equations of motion ).

Now it is straightforward to compute the canonical momentum corresponding to $\xi$

$$
\Pi_{\xi} \equiv J_{1}-J_{2}=\frac{\partial \mathcal{L}\left(\dot{\xi}, \xi^{\prime}\right)}{\partial \dot{\xi}}=\frac{1}{2 \pi \alpha^{\prime}} \frac{\sqrt{g_{1}}\left(g_{2}-4 g_{1}\right)}{e^{\Phi_{0} / 2}}(J+m) \omega
$$

Computing the energy we find

$$
E=\Pi_{\xi} \dot{\xi}-\mathcal{L}\left(\dot{\xi}, \xi^{\prime}\right)=\frac{1}{2 \pi \alpha^{\prime}}\left[\frac{\sqrt{g_{1}}\left(g_{2}-4 g_{1}\right)}{2 e^{\Phi_{0} / 2}}(J+m) \omega^{2}+e^{\Phi_{0} / 2} \sqrt{g_{1}}(J-m)\right],
$$

or

$$
E=\frac{1}{2}\left(J_{1}-J_{2}\right) \omega+\frac{1}{2 \pi \alpha^{\prime}} e^{\Phi_{0} / 2} \sqrt{g_{1}}(J-m),
$$

where

$$
\begin{gathered}
\sqrt{g_{1}}\left(g_{2}-4 g_{1}\right)=\frac{2 \sqrt{A} \sin ^{2}(\tilde{\theta} / 2)}{\cos (\tilde{\theta} / 2) \sqrt{1+\gamma^{2} A^{2} \sin ^{2} \tilde{\theta}}}, \\
\sqrt{g_{1}}=\frac{\sqrt{1+\gamma^{2} A^{2} \sin ^{2} \tilde{\theta}}}{2 \sqrt{A} \cos (\tilde{\theta} / 2)}, \quad g_{2}-4 g_{1}=\frac{4 A \sin ^{2}\left(\frac{\tilde{\theta}}{2}\right)}{1+\gamma^{2} A^{2} \sin ^{2} \tilde{\theta}}
\end{gathered}
$$

\section{Rotating strings with spins in the both cy- cles, $S^{2}$ and $S^{3}$}

In this section, the discussion follows closely the one in the $\gamma$-deformed $S^{5}$ part. If we put

$$
\psi=0, \quad r=0,
$$


the relevant parts of the metric and the B-field are

$$
\begin{gathered}
d s^{2}=-e^{\Phi_{0}} d t^{2}+A d(\tilde{\theta}+\theta)^{2}+ \\
+\frac{A}{1+\gamma^{2} A^{2} \sin ^{2}(\tilde{\theta}+\theta)}(d \varphi+\cos (\tilde{\theta}+\theta) d \tilde{\varphi})^{2}+\frac{A \sin ^{2}(\tilde{\theta}+\theta)}{1+\gamma^{2} A^{2} \sin ^{2}(\tilde{\theta}+\theta)} d \tilde{\varphi}^{2} \\
B_{2}=d \varphi \wedge d \tilde{\varphi}
\end{gathered}
$$

where $A=\frac{\alpha^{\prime} g_{s} N e^{\Phi_{0}}}{4}$.

The ansatz

$$
t=\tau ; \quad \theta=-\tilde{\theta} ; \quad \varphi=-m \sigma+\omega_{1} \tau ; \quad \tilde{\varphi}=m \sigma+\omega_{2} \tau,
$$

is a solution of the equations of motion ( $m$ is an integer).

We should also impose the Virasoro constraints in order to ensure conformal invariance. One of them is trivially satisfied by our ansatz and from the second one we obtain the following relation for $\omega_{1}+\omega_{2} \equiv \omega$ :

$$
\omega^{2}=\frac{e^{\Phi_{0}}}{A}, \quad \text { i.e. } \quad \omega=\frac{2}{\sqrt{\alpha^{\prime} g_{s} N}} .
$$

The relevant bosonic part of the classical string action depends on the metric $G_{M N}$ and the NS-NS 2-form B-field $B_{M N}$, i.e. is given by

$$
S=-\frac{1}{4 \pi} \int d \tau d \sigma\left[g^{p q} G_{M N} \partial_{p} X^{M} \partial_{q} X^{N}-\epsilon^{p q} B_{M N} \partial_{p} X^{M} \partial_{q} X^{N}\right]
$$

where $\epsilon^{01}=1$ and $g^{p q} \equiv \sqrt{-h} h^{p q}\left(h^{p q}\right.$ is a world-sheet metric with Minkowski signature, i.e. in the conformal gauge $\left.g^{p q}=\operatorname{diag}(-1,1)\right)$.

The Polyakov action for this ansatz is simply

$$
S=-\frac{1}{4 \pi} \int d \tau d \sigma\left[e^{\Phi_{0}} \dot{t}^{2}-A(\dot{\varphi}+\dot{\tilde{\varphi}})^{2}-2 m(\dot{\varphi}+\dot{\tilde{\varphi}})\right] .
$$

Now it is straightforward to compute the angular momenta $J_{1}$ and $J_{2}$ corresponding to $\varphi$ and $\tilde{\varphi}$

$$
J_{1}=J_{2}=A\left(\omega_{1}+\omega_{2}\right)+m=A \omega+m .
$$


Computing the energy we find

$$
E=e^{\Phi_{0}} .
$$

Using the relation (6.4) and set $A=\frac{\alpha^{\prime} g_{s} N e^{\Phi_{0}}}{4}$ we can rewrite the energy with the total spin $J=J_{1}+J_{2}=2 A \omega+2 m$ as follow

$$
E=\frac{(J-2 m) \omega}{2} .
$$

We should note also that the metrics (5.1) and (6.1) are identical. Changing $\tilde{\theta}+\theta$ by $\tilde{\theta}$ and $\varphi$ by $\psi$ in metric (6.1) we obtain the metric (5.1). Consequently, the ansatz

$$
t=\tau ; \quad \tilde{\theta}=0 ; \quad \psi=-m \sigma+\omega_{1} \tau ; \quad \tilde{\varphi}=m \sigma+\omega_{2} \tau,
$$

is a solution of the equations of motion for the string-frame (5.1). Therefore, the result for energy will be the same as in (6.9).

\section{More rotating strings}

Let us consider the following limiting case of the geometry considered in [6]. If in background (2.1) we take the limit $\theta, \tilde{\theta}, \varphi$ and $\tilde{\varphi} \longrightarrow 0$ the only contributions will come from the first and second terms in the square brackets. The resulting geometry then becomes:

$$
d s_{\text {limit }}^{2}=e^{\Phi(r)}\left[-d t^{2}+d X_{1}^{2}+d X_{2}^{2}+d Z^{2}+\alpha^{\prime} g_{s} N d r^{2}+\frac{\alpha^{\prime} g_{s} N}{4} d \psi^{2}\right] .
$$

The classical equations of motion for the string sigma model are:

$$
\begin{aligned}
& \partial_{\alpha}\left(e^{\Phi(r)} \eta^{\alpha \beta} \partial_{\beta} t\right)=0 \\
& \partial_{\alpha}\left(e^{\Phi(r)} \eta^{\alpha \beta} \partial_{\beta} X^{i}\right)=0 \\
& \partial_{\alpha}\left(e^{\Phi(r)} \eta^{\alpha \beta} \partial_{\beta} r\right)=\frac{1}{2}\left(e^{\Phi(r)}\right) \eta^{\alpha \beta}\left[-\partial_{\alpha} t \partial_{\beta} t+\partial_{a} X^{i} \partial_{b} X^{i}\right]
\end{aligned}
$$

We make the following ansatz:

$$
\begin{aligned}
& t=\kappa \tau \\
& X_{i}=x_{i}(\tau) \rho_{i}(\sigma) \\
& r=r(\sigma)
\end{aligned}
$$


The equations of motion for the $X_{i}$ variables $(7.2,7.3,7.4)$ then become

$$
-e^{\Phi(r)} \ddot{x}_{i}(\tau)+\rho_{i}(\sigma)+x_{i}(\tau) \partial_{\sigma}\left(e^{\Phi(r)}\right)=0
$$

We can now impose natural separation of variables which leads to

$$
\begin{aligned}
& \ddot{x}_{i}(\tau)+\omega_{1}^{2} x_{i}(\tau)=0 \\
& \partial_{\sigma}\left(e^{\Phi(r)} r h o^{\prime}(\sigma)\right)+\omega_{1}^{2} e^{\Phi(r)} \rho^{\prime}(\sigma)=0 .
\end{aligned}
$$

The solutions of (7.7) are correspondingly

$$
x_{1}=\cos \omega \tau, \quad x_{2}=\sin \omega \tau
$$

For the radial variable $r$ we get

$$
\begin{array}{r}
\partial_{\sigma}\left(e^{\Phi(r)} \partial_{\sigma} r\right)=\frac{1}{2} \partial_{r} e^{\Phi(r)}\left[\kappa^{2}-\omega_{1}^{2} \rho_{1}^{2} \sin ^{2} \omega \tau-\omega_{1}^{2} \rho_{2}^{2} \cos ^{2} \omega \tau\right. \\
\left.+\rho_{1}^{\prime 2} \cos ^{2} \omega \tau+{\rho_{2}^{\prime}}^{2} \sin ^{2} \omega \tau\right]
\end{array}
$$

The Virasoro constraints can be written in the form

$$
\begin{aligned}
& e^{\Phi(r)} r^{2}+e^{\Phi(r)}\left[-\kappa^{2}+\omega_{1}^{2} \rho_{1}^{2} \sin ^{2} \omega_{1} \tau\right. \\
& \left.\omega_{1}^{2} \rho_{2}^{2} \cos ^{2} \omega \tau+\rho_{1}^{\prime 2} \cos ^{2} \omega \tau+\rho_{2}^{\prime 2} \sin ^{2} \omega \tau\right]=0 .
\end{aligned}
$$

One can write now the corresponding conserved charges

$$
\begin{gathered}
E=\frac{\kappa}{2 \pi \alpha^{\prime}} \int e^{\Phi(r)} d \sigma \\
J=\frac{1}{2 \pi \alpha^{\prime}} \int e^{\Phi(r)} \rho_{1} \rho_{2}\left[x_{1} \partial_{\tau} x_{2}-x_{2} \partial_{\tau} x_{1}\right] d \sigma \\
=\frac{\omega}{2 \pi \alpha^{\prime}} \int e^{\Phi(r)} \rho_{1} \rho_{2} d \sigma
\end{gathered}
$$

To solve the equations of motion we impose the following simplifying condition

$$
\rho_{1}=\rho_{2}=\rho
$$

after which the equations of motion become

$$
\partial_{\sigma}\left(e^{\Phi(r)} \partial_{\sigma} r\right)-\frac{1}{2} \partial_{r} e^{\Phi(r)}\left[\kappa^{2}-\omega_{1}^{2} \rho^{2}+{\rho^{\prime}}^{2}\right]
$$


The Virasoro constraints take the form

$$
e^{\Phi(r)}{r^{\prime}}^{2}+e^{\Phi(r)}\left[-\kappa^{2}+\omega_{1}^{2} \rho^{2}+{\rho^{\prime}}^{2}\right]=0 .
$$

which gives for the angular momentum the expression

$$
J=\frac{\omega}{2 \pi \alpha^{\prime}} \int e^{\Phi(r)} \rho^{2} d \sigma .
$$

The AdS/CFT duality teach us that the corresponding state in the gauge theory has the same quantum numbers. In order the semiclassical approximation to be valid the action should be large and thus we are dealing with a state in the IR region of the corresponding gauge theory with large momentum $J$ and energy $E$. One can consider now the string at the stationary point $r_{0}=0$. The equation of motion for $r$ then is trivially satisfied and the Virasoro constraints gives an equation for $\rho$

$$
\rho^{\prime 2}+\omega_{1}^{2} \rho^{2}=\kappa^{2} .
$$

The solution is

$$
\rho(\sigma)=A \sin \omega \sigma,
$$

and using again the Virasoro constraint we get for $A$

$$
A=\frac{\kappa}{\omega} .
$$

The expression for the energy and angular momentum then become

$$
E=2 \pi \kappa T_{s} ; \quad J=\frac{\kappa^{2}}{\omega_{1}^{2}} \pi T_{s},
$$

where $T_{s}$ is the string tension. It is obvious that we reproduce the well known Regge relation

$$
J=\frac{1}{4 \pi T_{s, e f f}} E^{2} .
$$

One can study now the fluctuations around the given configuration and find the quantum corrections to the relation we obtained, but this is subject to another investigation. The main lesson we can extract is that having obtained a solution independent of $\gamma$, we reproduce the well known Regge behavior and one can conclude the our solution is physically reasonable. Although our 
result do not give conclusive solution of the decoupling of the Kaluza-Klein modes, it gives more arguments to the conjecture that $\gamma$ deformation lifts the KK-modes and one can serve as a mechasnism of separating the physical solutions.

As another test of the conjecture we consider strings with $r$ nontrivially depending on the worldsheet coordinate $\sigma$, i.e. the string is extended in $r$ direction too.

The Virasoro constraint and the equation of motion for $\rho(\sigma)$ are:

$$
\begin{gathered}
A r^{\prime 2}(\tilde{\sigma})+\rho^{\prime 2}(\tilde{\sigma})+\rho^{2}(\tilde{\sigma})=\frac{B^{2}}{\omega_{1}^{2}}, \\
\rho^{\prime \prime}(\tilde{\sigma})+\frac{d \Phi(r)}{d r} r^{\prime}(\tilde{\sigma}) \rho^{\prime}(\tilde{\sigma})+\rho(\tilde{\sigma})=0,
\end{gathered}
$$

where $\tilde{\sigma}=\omega_{1} \sigma$ and $A=\alpha^{\prime} g_{s} N, B^{2}=\kappa^{2}-\frac{\alpha^{\prime} g_{s} N}{4} \omega_{2}^{2}$.

For small $\rho$ we have:

$$
\begin{gathered}
A r^{\prime 2}(\tilde{\sigma})+\rho^{\prime 2}(\tilde{\sigma})=\frac{B^{2}}{\omega_{1}^{2}}, \\
\rho^{\prime \prime}(\tilde{\sigma})+\frac{d \Phi(r)}{d \tilde{\sigma}} \rho^{\prime}(\tilde{\sigma})=0 .
\end{gathered}
$$

Integrating equation (7.24) once we obtain:

$$
\left|\rho^{\prime}(\tilde{\sigma})\right|=C e^{-\Phi(r)}
$$

For $r$ near by to fixed $r_{0}$ we have the following approximation

$$
\rho^{\prime}(\tilde{\sigma})=-C e^{-\Phi\left(r_{0}\right)}\left[1-\frac{d \Phi\left(r_{0}\right)}{d r}\left(r-r_{0}\right)\right] .
$$

Using the Virasoro constraint (7.23) we obtain for $r(\tilde{\sigma})$ the equation:

$$
A r^{\prime 2}(\tilde{\sigma})=\frac{B^{2}}{\omega_{1}^{2}}\left[1-\frac{C^{2} \omega_{1}^{2}}{B^{2}} e^{-2 \Phi\left(r_{0}\right)}\left(1-\frac{d \Phi\left(r_{0}\right)}{d r}\left(r-r_{0}\right)\right)^{2}\right]
$$

when $r_{1}^{\star} \leq r \leq r_{2}^{\star}$, where

$$
r_{1}^{\star}=r_{0}-\frac{\frac{B e^{\Phi\left(r_{0}\right)}}{C \omega_{1}}-1}{\frac{d \Phi\left(r_{0}\right)}{d r}}, \quad r_{2}^{\star}=r_{0}+\frac{\frac{B e^{\Phi\left(r_{0}\right)}}{C \omega_{1}}+1}{\frac{d \Phi\left(r_{0}\right)}{d r}},
$$


are the turning points of string and $r_{0}+\frac{1}{\frac{d \Phi\left(r_{0}\right)}{d r}}$ is the center of mass. This equation can be rewritten as:

$$
\frac{d r}{\sqrt{1-\frac{C^{2} \omega_{1}^{2}}{B^{2}} e^{-2 \Phi\left(r_{0}\right)}\left(1-\frac{d \Phi\left(r_{0}\right)}{d r}\left(r-r_{0}\right)\right)^{2}}}=\frac{B}{\omega_{1} \sqrt{A}} d \tilde{\sigma}
$$

This relation is appropriate for the computation of the conserved charges in our problem (the energy and angular momenta ).

The $2 \pi$ periodic solutions of (7.27) and (7.26) are:

$$
\begin{gathered}
r(\sigma)=r_{0}+\frac{1}{\frac{d \Phi\left(r_{0}\right)}{d r}}\left[1+\frac{B e^{\Phi\left(r_{0}\right)}}{C \omega_{1}} \sin (m \sigma)\right], \\
\rho(\sigma)=\frac{B \sqrt{A} e^{\Phi\left(r_{0}\right)}}{C \omega_{1}} \frac{1}{\frac{d \Phi\left(r_{0}\right)}{d r}}[1-\cos (m \sigma)],
\end{gathered}
$$

where $m=\frac{C}{\sqrt{A}} e^{-\Phi\left(r_{0}\right)} \frac{d \Phi\left(r_{0}\right)}{d r} \omega_{1}=4 k+1, \quad k=0,1,2,3, \ldots$.

For energy and spins in this case we have:

$$
\begin{gathered}
E=2 \kappa \frac{\sqrt{A} e^{2 \Phi\left(r_{0}\right)}}{C \frac{d \Phi\left(r_{0}\right)}{d r}} \frac{1}{\omega_{1}}\left[1+\frac{B e^{\Phi\left(r_{0}\right)}}{\pi C} \frac{1}{\omega_{1}}\right] \\
J_{2}=\frac{A^{3 / 2} e^{2 \Phi\left(r_{0}\right)}}{2 C \frac{d \Phi\left(r_{0}\right)}{d r}} \frac{\omega_{2}}{\omega_{1}}\left[1+\frac{B e^{\Phi\left(r_{0}\right)}}{\pi C} \frac{1}{\omega_{1}}\right], \\
J_{1}=\frac{2 A B^{2} e^{3 \Phi\left(r_{0}\right)}}{\pi C^{2}\left(\frac{d \Phi\left(r_{0}\right)}{d r}\right)^{2}} \frac{1}{\omega_{1}}\left[\frac{3 \pi}{2}-4 \frac{\sqrt{A} e^{\Phi\left(r_{0}\right)}}{C \frac{d \Phi\left(r_{0}\right)}{d r}} \frac{1}{\omega_{1}}+\frac{1}{3} \frac{B \sqrt{A} e^{2 \Phi\left(r_{0}\right)}}{C^{2} \frac{d \Phi\left(r_{0}\right)}{d r}} \frac{1}{\omega_{1}^{2}}\right] .
\end{gathered}
$$

As a result of our considerations one can conclude that there exsist indeed physical solutions that give the conserved quantities independent of the deformation parameter. Once again, we point out that these computations show that the conjecture can serve as a mechanism of separating physically relevant solutions. It is important however to point out that sometimes the background can "cheate" and one can have a realization of some "secrete" mechanism of cancelation of the contributions coming from gamma deformation with KK modes still in the game and the conjecture we checked in this paper cannot be considered as a definite way of separating pure gauge theory effects. We will discusse more on this issue in the next Section. 


\section{Conclusions}

In this paper we considered string theory in the deformed Maldacena -Nunez background studied in [6] at supergravity level. We studied various string configurations of string rotating in several cycles of the geometry and pulsating strings as well, and discussed the effect of the deformation.

After listing the basic formulae for the deformed Maldacena-Nunez background in Section 2, in Section 3 we present the analysis of the simple rotating strings in the $S^{2} \times \mathbb{R}$. Since the calculations cannot be given in analytic form, we consider the limit of short and long strings. In this approximation we show that the energy increases due to the deformation parameter $\gamma$. We arrived to the same conclusions in Section 4 where we consider short and long pulsating strings in the same cycle of the geometry. Since the geometry have one more cycle in its five dimensional part, we consider fast moving strings in $S^{3} \times \mathbb{R}$. Detailed consideration show that again we have increasing of the energy due to the deformation.

In [6] it was conjectured that if one can separate a sector in which the gamma deformation completely decouples, one can state that the effect should be purely due to the gauge theory with no contributions from KK modes. In the last section we were able to find a nontrivial string configurations in which this can be achieved.

This check is quite remarkable, so let us comment in some details what we found. As we mentioned above, the conjecture made in [6] is that the gammadeformed and the undeformed background only differ in the dynamics of the KK modes. To check the conjecture we considered various rotating and pulsating string configurations. The first type of configurations is when strings rotate in any combinations of $\varphi$ and $\tilde{\varphi}$ directions. By means of AdS/CFT correspondence these should describe long operators in the gauge theory of the form

$$
\mathcal{A}_{K K}=\mathcal{O O} \cdots \mathcal{O K K K \mathcal { K }} \cdots \mathcal{O}
$$

where $\mathcal{O}$ represents any $\mathcal{N}=1 \mathrm{SYM}$ operator and $\mathcal{K}$ - any operator made out of KK modes. The second type configurations is when strings do not rotate in those directions. The statement of the conjecture is that in this case the long operators are made out of operators of the SYM fields

$$
\mathcal{A}=\mathcal{O O} \cdots \mathcal{O O} \cdots \mathcal{O}
$$

What are the reasons for the above comments? First of all, let us mention 
the dual Lagrangian of the Maldacena-Nunez model

$$
\mathcal{L}=F_{\mu \nu}^{2}+\lambda D \lambda+\mathcal{L}_{K K},
$$

where $F_{\mu \nu}^{2}$ is the usual YM curvature and $\lambda$ is the corresponding majorana spinor. The last term $\mathcal{L}_{K K}$ is the lagrangian describing the KK modes and is given by (for recent discussion see [56])

$$
\mathcal{L}_{K K}=(D \phi)^{2}+\Psi D \Psi+V(\lambda, \Psi, \phi),
$$

where $\phi$ is a scalar, $\Psi$ is a spinor and $V$ is a potential whose form is not important in this discussion. So, what Maldacena and Nunez have done is actually to write a background that represents the strong coupling regime of the lagrangian

$$
\mathcal{L}=F_{\mu \nu}^{2}+\lambda D \lambda+(D \phi)^{2}+\Psi D \Psi+V(\lambda, \Psi, \phi)
$$

which is nothing but an UV completion of $\mathcal{N}=1$ SYM.

Let us turn now to what is done in [6]. In this paper Gursoy and Nunez provided a continuous set of UV completions parameterized by $\gamma$. For each value of this parameter we have difference in the dynamics of the KK modes due to the changes in the potential $V(\phi, \lambda, \Psi)$ by a dipole deformation. The conclusions from our computations can be put in two different, complementary and physically nice ways:

1) In the first type of configurations the strings charged under $U(1) \times$ $U(1)$ do experience changes in their spectrum and dynamical relations $E=$ $E\left(J_{1}, J_{2}\right)$ as the background is deformed. Hence, those strings "remember" that they are composed out of a large number of KK modes (which are charged under $U(1) \times U(1)$ and hence the $\gamma$ deformation changes its dynamics ) and also SYM operators; these are strings of the form (8.1) above. Strings like the one in section 7 (which are of the form (8.2)) are made out of only SYM operators. Hence they do not see changes in the deformed background.

2) Our considerations show that the Gursoy-Nunez backgrounds do indeed provide different UV completions and that the different completions are labelled by a continuous parameter $\gamma$. The different values of $\gamma$ does not change everything, but just the UV completion that the supergravity solution is giving to $\mathcal{N}=1 \mathrm{SYM}$.

Giving support to the Gursoy-Nunez conjecture a reasonable question arises: how to decouple completely the UV completion? Certainly this is 
an open question, because it is not known how to calculate string theory in RR backgrounds. What will happen if such a technique is available? One can speculate that the KK modes will be made infinitely heavy and the spectrum will be just that of SYM. Then our rotating strings that do depend on $\varphi$ and $\tilde{\varphi}$ upon quantization will become very heavy also. The present situation however do not alow all that and we have to live with the KK modes. Since the mass of these KK modes is similar to the scale of confinement, the relevant and important problem is to decide whether or not the background we consider is giving us a result we can trust or not.

As a result of the above discussion, we see that the proposal of Gursoy and Nunez is to gamma deform the background (which has completely different behavior in the UV) and compute the same things in both backgrounds. If the results depend on $\gamma$ in the deformed background, the result contains effects due to KK modes, if not - this is purely gauge theory effects. Our considerations of rotating strings in different directions, in our opinion, are giving a very remarkable check of all this picture described above.

As we pointed out in the previous Section, sometimes it happens that KK modes can cancel in some way the gamma deformations and one can end up with the same result but with KK modes not really decoupled. Certainly the idea of using gamma deformation of such backgrounds to separate the contributions from KK modes from the pure gauge theory effects deserves further study and we will return to this issue in future investigations.

Acknowledgments We would like to thank Carlos Nunez for suggesting this problem to us and for his valuable comments. The work of N.P.B. was partially supported by an EVRIKA foundation educational award.

\section{Appendix: A circular string in the back- ground (7.1).}

Let us consider the following circular string ansatz ${ }^{5}$ :

$$
\begin{array}{ll}
x=\rho \cos \left(\omega_{1} \tau+\alpha(\sigma)\right) ; & \psi=\omega_{2} \tau+\beta(\sigma) ; t=\kappa \tau \\
y=\rho \sin \left(\omega_{1} \tau+\alpha(\sigma)\right) ; \quad \rho=\text { const } ; \quad r=r(\sigma) .
\end{array}
$$

\footnotetext{
${ }^{5}$ In this appendix we use the notations $X_{1}=x, X_{2}=y$.
} 
The Virasoro constraints for this ansatz are:

$$
\begin{gathered}
\rho^{2} \omega_{1} \alpha^{\prime}(\sigma)+\frac{\alpha^{\prime} g_{s} N}{4} \omega_{2} \beta^{\prime}(\sigma)=0 \\
\rho^{2} \alpha^{\prime 2}(\sigma)+\frac{\alpha^{\prime} g_{s} N}{4} \beta^{\prime 2}(\sigma)+\alpha^{\prime} g_{s} N r^{\prime 2}(\sigma)+\rho^{2} \omega_{1}^{2}+\frac{\alpha^{\prime} g_{s} N}{4} \omega_{2}^{2}-\kappa^{2}=0, \\
\text { or } \quad\left(\rho^{2}+\frac{4 \rho^{4}}{A} \frac{\omega_{1}^{2}}{\omega_{2}^{2}}\right) \alpha^{\prime 2}(\sigma)+A r^{\prime 2}(\sigma)=B
\end{gathered}
$$

where

$$
A=\alpha^{\prime} g_{s} N, \quad B=\kappa^{2}-\rho^{2} \omega_{1}^{2}-\frac{A}{4} \omega_{2}^{2}>0 .
$$

The induced world-sheet matric with Virasoro constraints taken into account has the form:

$$
d s_{w s}^{2}=e^{\Phi(r(\sigma))} B\left(-d \tau^{2}+d \sigma^{2}\right)
$$

and then we have

$$
\sqrt{-g} g^{p q} \equiv \gamma^{p q}=\operatorname{diag}(-1,1) .
$$

The relevant Polyakov action for this geometry is:

$$
\begin{aligned}
S=-\frac{1}{4 \pi} \int d \tau d \sigma e^{\Phi(r(\sigma))}\left[\kappa^{2}-\right. & \rho^{2} \omega_{1}^{2}-\frac{A}{4} \omega_{2}^{2}+ \\
& \left.+\rho^{2} \alpha^{\prime 2}(\sigma)+\frac{A}{4} \beta^{\prime 2}(\sigma)+A r^{\prime 2}(\sigma)\right] .
\end{aligned}
$$

The equations of motion for $\alpha(\sigma)$ and $\beta(\sigma)$ are the identical:

$$
\begin{aligned}
& \alpha^{\prime \prime}(\sigma)+\frac{d \Phi(r)}{d r} r^{\prime}(\sigma) \alpha^{\prime}(\sigma)=0, \\
& \beta^{\prime \prime}(\sigma)+\frac{d \Phi(r)}{d r} r^{\prime}(\sigma) \beta^{\prime}(\sigma)=0 .
\end{aligned}
$$

Integrating them once we obtain

$$
\begin{array}{r}
\frac{d}{d \sigma} \alpha^{\prime 2}(\sigma)+2 \frac{d}{d \sigma} \Phi(r) \alpha^{\prime 2}(\sigma)=0 \\
\alpha^{\prime 2}(\sigma)=C_{1} e^{-2 \Phi(r)}, \\
\beta^{\prime 2}(\sigma)=C_{2} e^{-2 \Phi(r)} .
\end{array}
$$


One can check that the Virasoro constraint (9.2) are satisfied.

For $r(\sigma)$ we have the following equation of motion:

$$
2 A r^{\prime \prime}(\sigma)+A \frac{d \Phi(r)}{d r} r^{\prime 2}(\sigma)-\frac{d \Phi(r)}{d r}\left[B+\rho^{2} \alpha^{\prime 2}(\sigma)+\frac{A}{4} \beta^{\prime 2}(\sigma)\right]=0 .
$$

Using the Virasoro constraint (9.3) we obtain for $r(\sigma)$ the equation

$$
A r^{\prime \prime}(\sigma)+A \frac{d \Phi(r)}{d r} r^{\prime 2}(\sigma)-\frac{d \Phi(r)}{d r} B=0
$$

One can easily integrate this equation as follow:

$$
\begin{gathered}
A \frac{d}{d \sigma} r^{\prime 2}(\sigma)+2 \frac{d \Phi(r)}{d r} r^{\prime}(\sigma)\left[A r^{\prime 2}(\sigma)-B\right]=0 \\
\frac{d}{d \sigma}\left[A r^{\prime 2}(\sigma)-B\right]+2\left[A r^{\prime 2}(\sigma)-B\right] \frac{d}{d \sigma} \Phi(r)=0 \\
\frac{1}{\left[A r^{\prime 2}(\sigma)-B\right]} \frac{d}{d \sigma}\left[A r^{\prime 2}(\sigma)-B\right]=-2 \frac{d}{d \sigma} \Phi(r) \\
\left|A r^{\prime 2}(\sigma)-B\right|=C e^{-2 \Phi(r)}
\end{gathered}
$$

In order the resulting solution to be periodic in $\sigma$ one should take the constant $C$ negative. At the end of the day we find

$$
r^{\prime 2}(\sigma)=\frac{B}{A}-\frac{C}{A} e^{-2 \Phi(r)}
$$

where $\frac{B}{C}<e^{-2 \Phi_{0}}$. This equation can be rewritten as:

$$
d \sigma=\frac{1}{\sqrt{\frac{B}{A}-\frac{C}{A} e^{-2 \Phi(r)}}} d r
$$

This relation is useful for the computation of the conserved charges in our problem (the energy and angular momenta ) which can be written in the 
form:

$$
\begin{array}{r}
E=\frac{\kappa}{2 \pi} \int_{0}^{2 \pi} e^{\Phi(r)} d \sigma, \\
J_{1}=\frac{\rho^{2} \omega_{1}}{2 \pi} \int_{0}^{2 \pi} e^{\Phi(r)} d \sigma, \\
J_{2}=\frac{(A / 4) \omega_{2}}{2 \pi} \int_{0}^{2 \pi} e^{\Phi(r)} d \sigma .
\end{array}
$$

Therefore, the final expression for the energy takes the form

$$
E=\frac{\kappa}{\rho^{2} \omega_{1}+\frac{A}{4} \omega_{2}}\left(J_{1}+J_{2}\right) .
$$

We want to emphasize that this result is exact and does not depend on the deformation parameter.

To complete the analysis, we will present here the cases of the limits of short and long strings. In the first case we have $r \longrightarrow 0$. In this limit the dilaton field has the following behavior:

$$
e^{-2 \Phi(r)} \approx e^{-2 \Phi_{0}}\left[1-\frac{8}{9} r^{2}+\frac{224}{405} r^{4}\right] .
$$

Substituting this approximation into the equation (9.15) we get:

$$
r^{\prime 2}(\sigma)=\frac{B}{A}-\frac{C}{A} e^{-2 \Phi_{0}}\left[1-\frac{8}{9} r^{2}+\frac{224}{405} r^{4}\right]
$$

or

$$
r^{\prime 2}(\sigma)=\frac{224}{405} \frac{C}{A} e^{-2 \Phi_{0}}\left(r_{2}^{2}-r^{2}\right)\left(r^{2}-r_{1}^{2}\right),
$$

where

$$
\begin{aligned}
& r_{1}^{2}=\frac{45}{56}\left[1-\frac{3}{\sqrt{5}} \sqrt{\frac{14}{9} \frac{B}{C} e^{2 \Phi_{0}}-1}\right]<1, \\
& r_{2}^{2}=\frac{45}{56}\left[1+\frac{3}{\sqrt{5}} \sqrt{\frac{14}{9} \frac{B}{C} e^{2 \Phi_{0}}-1}\right]<1
\end{aligned}
$$


are the turning points of the short string, $0<r_{1}^{2}<r^{2}<r_{2}^{2}<1$ and $\frac{9}{14}<\frac{B}{C} e^{2 \Phi_{0}}<1$.

The $2 \pi$ periodic solution of $(9.23)$ is:

$$
r(\sigma)=r_{2} \mathbf{d n}\left[\frac{\mathbf{K}}{\pi}\left(\frac{\pi}{2}-\sigma\right) \mid \frac{r_{2}^{2}-r_{1}^{2}}{r_{2}^{2}}\right],
$$

where $C=\left(\frac{9 \mathrm{~K}}{4 \pi r_{2}}\right)^{2} \frac{5}{14} A e^{2 \Phi_{0}}$.

For energy and spins in short string case we have:

$$
\begin{gathered}
E=\frac{\kappa}{2 \pi} \int_{0}^{2 \pi} e^{\Phi(r)} d \sigma=2 \frac{\kappa}{2 \pi} \int_{r_{1}}^{r_{2}} \frac{e^{\Phi(r)}}{\sqrt{\frac{B}{A}-\frac{C}{A} e^{-2 \Phi(r)}}} d r \approx \\
\approx \frac{\kappa r_{2} e^{\Phi_{0}}}{\mathbf{K}} \int_{r_{1}}^{r_{2}} \frac{1+\frac{4}{9} r^{2}+\frac{8}{405} r^{4}}{\sqrt{\left(r_{2}^{2}-r^{2}\right)\left(r^{2}-r_{1}^{2}\right)}} d r= \\
=e^{\Phi_{0}} \kappa+\frac{4}{9} e^{\Phi_{0}} \kappa r_{2}^{2} \frac{\mathbf{E}}{\mathbf{K}}+\frac{8}{405} \frac{e^{\Phi_{0}} \kappa r_{2}^{2}}{3 \mathbf{K}}\left[2\left(r_{1}^{2}+r_{2}^{2}\right) \mathbf{E}-r_{1}^{2} \mathbf{K}\right], \\
J_{1}=e^{\Phi_{0}} \rho^{2} \omega_{1}\left[1+\frac{4}{9} r_{2}^{2} \frac{\mathbf{E}}{\mathbf{K}}+\frac{8}{405} \frac{r_{2}^{2}}{3 \mathbf{K}}\left[2\left(r_{1}^{2}+r_{2}^{2}\right) \mathbf{E}-r_{1}^{2} \mathbf{K}\right]\right] \\
J_{2}=e^{\Phi_{0}} \frac{A}{4} \omega_{2}\left[1+\frac{4}{9} r_{2}^{2} \frac{\mathbf{E}}{\mathbf{K}}+\frac{8}{405} \frac{r_{2}^{2}}{3 \mathbf{K}}\left[2\left(r_{1}^{2}+r_{2}^{2}\right) \mathbf{E}-r_{1}^{2} \mathbf{K}\right]\right]
\end{gathered}
$$

In long string case (i.e. $r \longrightarrow \infty$ ) the dilaton field has the following behavior:

$$
e^{-2 \Phi(r)} \approx \frac{4 r^{1 / 2}}{e^{2 r}} .
$$

Using this approximation in the equation (9.15)we get

$$
r^{\prime 2}(\sigma)=\frac{B}{A}-\frac{C}{A} \frac{4 r^{1 / 2}}{e^{2 r}} .
$$

The turning point $r_{0}$ satisfies the equality

$$
\frac{4 r_{0}^{1 / 2}}{e^{2 r_{0}}}=\frac{B}{C}
$$


For energy and spins we find:

$$
\begin{gathered}
E=\frac{\kappa}{\pi} \sqrt{\frac{A}{C}} \int_{0}^{r_{0}} \frac{e^{r} r^{-1 / 4}}{\sqrt{\frac{B}{C}-\frac{4 r^{1 / 2}}{e^{2 r}}}} d r \approx \\
\approx \frac{\kappa}{\pi} \sqrt{\frac{A}{B}} r_{0}^{3 / 4} \sqrt{2} \frac{\Gamma(1 / 2) \Gamma(1)}{\Gamma(3 / 2)}{ }_{1} \mathrm{~F}_{1}\left[1,3 / 2, r_{0}\right] \approx \frac{\kappa}{\sqrt{\pi}} \sqrt{\frac{A}{B}} \sqrt{2} r_{0}^{1 / 4} e^{r_{0}}, \\
J_{1} \approx \frac{\rho^{2} \omega_{1}}{\sqrt{\pi}} \sqrt{\frac{A}{B}} \sqrt{2} r_{0}^{1 / 4} e^{r_{0}} \\
J_{2} \approx \frac{A \omega_{2}}{4 \sqrt{\pi}} \sqrt{\frac{A}{B}} \sqrt{2} r_{0}^{1 / 4} e^{r_{0}} .
\end{gathered}
$$

The expressions we found for the limiting cases of short and long strings are consistent with the general result (9.20) we already found.

The string configurations we studied in this Appendix are somhow trivial. This i because the circular string is "spinning into itselfs" and looks like point particle circling on the circle with radius $\rho$. Nevertheless, it is good to have explicit solutions supporting the conjecture of separating the KK modes.

At the end, one can conclude from the considerations in this Appendix that there exist cases in which the KK modes are lifted by the deformation of the geometry and the obtained results are purely gauge theory effects.

\section{References}

[1] J.M. Maldacena, "The Large $\mathcal{N}$ Limit of Superconformal Field Theories and Supergravity", Adv.Theor.Math.Phys. 2 (1998) 231-252; Int.J.Theor.Phys. 38 (1999) 1113-1133, hep-th/ 9711200; E. Witten, "Anti-de Sitter space and holography", Adv. Theor. Math. Phys. 2, 253 (1998), hep-th/9802150; S. S. Gubser, I. R. Klebanov and A. M. Polyakov, "Gauge theory correlators from non-critical string theory", Phys. Lett. B428, 105 (1998), hep-th/9802109.

[2] D. Berenstein, J. M. Maldacena and H. Nastase, "Strings in flat space and pp waves from $\mathcal{N}=4$ Super Yang Mills", JHEP 0204, 013 (2002), hep-th/0202021. 
[3] S. S. Gubser, I. R. Klebanov and A. M. Polyakov, "A semi-classical limit of the gauge/string correspondence", Nucl. Phys. B 636, 99 (2002) hep-th/0204051.

[4] O. Lunin and J. Maldacena, "Deforming field theories with $\mathrm{U}(1) \mathrm{x} \mathrm{U}(1)$ global symmetry and their gravity duals", hep-th/0502086.

[5] R. G. Leigh and M. J. Strassler, "Exactly marginal operators and duality in fourdimensional $\mathcal{N}=1$ supersymmetric gauge theory", Nucl. Phys. B 447, 95 (1995) hep-th/9503121.

[6] U. Gursoy and C. Nunez, "Dipole deformations of $\mathcal{N}=1$ SYM and supergravity backgrounds with $U(1) \times U(1)$ global symmetry", hepth/0505100.

[7] J. M. Maldacena and C. Nunez, Phys. Rev. Lett. 86, 588 (2001) hepth/0008001.

[8] Changhyun Ahn, Justin F. Vazquez-Poritz, "Deformations of flows from type IIB supergravity", hep-th/0508075.

[9] A. Bergman and O. J. Ganor, JHEP 0010, 018 (2000) hep-th/0008030; A. Bergman, K. Dasgupta, O. J. Ganor, J. L. Karczmarek and G. Rajesh, Phys. Rev. D 65, 066005 (2002) hep-th/0103090.

[10] S. S. Pal, " $\beta$-deformations, potentials and KK modes" Phys. Rev. D 72, 065006 (2005), hep-th/0505257.

[11] S. Frolov, "Lax pair for strings in Lunin-Maldacena background", hepth/0503201.

[12] S. Frolov, R. Roiban, A. Tseytlin, "Gauge-string duality for superconformal deformations of $\mathcal{N}=4$ Super Yang-Mills theory", hep-th/0503192.

[13] S. A. Frolov, R. Roiban and A. A. Tseytlin, "Gauge-string duality for (non)supersymmetric deformations of $\mathrm{N}=4$ super Yang-Mills theory", hep-th/0507021.

[14] N. P. Bobev, H. Dimov and R. C. Rashkov, "Semiclassical strings in Lunin- Maldacena background", hep-th/0506063. 
[15] V. Niarchos and N. Prezas, "BMN Operators for $\mathcal{N}=1$ superconformal Yang-Mills theories and associated string backgrounds" JHEP 0306, 015 (2003), hep-th/0212111; R. de Mello Koch, J. Murugan, J. Smolic and M. Smolic, "Deformed PP-waves from the Lunin-Maldacena Background", hep-th/0505227; T. Mateos, "Marginal deformations of $\mathcal{N}=4$ SYM and Penrose limit with continuum spectrum", hep-th/0505243.

[16] N. Beisert and R. Roiban, "Beauty and the twist: The Bethe ansatz for twisted N = 4 SYM", arXiv:hep-th/0505187.

[17] Jorge G. Russo, "String Spectrum of Curved String Backgrounds Obtained by T-Duality and Shifts of Polar Angles", hep-th/0508125.

[18] A. Mauri, S. Penati, A. Santambrogio, D. Zanon, Exact Results in Planar N=1 Superconformal Yang-Mills Theory, hep-th/0507282; S. Penati, A. Santambrogio, D. Zanon, Two-Point Correlators in the BetaDeformed N=4 SYM at the next-to-leading Order, hep-th/0506150; D.Z. Freedman, Umut Gursoy, Comments on the Beta-Deformed N=4 SYM Theory, hep-th/0506128; G.C. Rossi, E. Sokatchev and Ya.S. Stanev, hep-th/0507113; S.M. Kuzenko, A.A. Tseytlin, "Effective Action of Beta-Deformed N=4 SYM Theory and ADS/CFT", hep-th/0508098.

[19] R. de Mello Koch, N. Ives, J. Smolic and M. Smolic, "Unstable giants", hep-th/0509007; R. C. Rashkov, K. S. Viswanathan and Y. Yang,"Ts...sT transformation on AdS(5) x S**5 background", hepth/0509058; S. Ryang, "Rotating Strings with Two Unequal Spins in Lunin-Maldacena Background", hep-th/0509195; A. H. Prinsloo, " $\gamma_{i^{-}}$ Deformed Lax pair for rotating strings in the fast motion limit", hepth/0510095; R. Hernandez, K. Sfetsos and D. Zoakos," Gravity duals for the Coulomb branch of marginally deformed $\mathrm{N}=4$ Yang-Mills", hepth/0510132; W. H. Huang, "Multi-spin string solutions in magnetic-flux deformed AdS(n) x S**m spacetime", hep-th/0510136, H. Y. Chen and S. P. Kumar, "Precision test of AdS/CFT in Lunin-Maldacena background", arXiv:hep-th/0511164.

[20] J. M. Pons and P. Talavera, "Semi-classical string solutions for $\mathcal{N}=1$ SYM", Nucl. Phys. B 665, 129 (2003) hep-th/0301178.

[21] S. Frolov and A. A. Tseytlin, "Semiclassical quantization of rotating superstring in $A d S_{5} \times S^{5}$ " JHEP 0206, 007 (2002), hep-th/0204226. 
[22] S. Frolov and A.A. Tseytlin, "Rotating string solutions: AdS/CFT duality in non-supersymmetric sectors", Phys. Lett. B 570, 96 (2003), hepth/0306143.

[23] S. Frolov and A. A. Tseytlin, "Multi-spin string solutions in $A d S_{5} \times S^{5}$ ", Nucl. Phys. B 668, 77 (2003), hep-th/0304255.

[24] A.A. Tseytlin, "Spinning strings and AdS/CFT duality", hepth/0311139; A. A. Tseytlin, "Semiclassical strings and AdS/CFT", in: Proceedings of NATO Advanced Study Institute and EC Summer School on String Theory: from Gauge Interactions to Cosmology, Cargese, France, 7-19 Jun 2004. hep-th/0409296.

[25] G. Arutyunov, J. Russo and A. A. Tseytlin, "Spinning strings in $A d S_{5} \times$ $S^{5}$ : New integrable system relations", Phys. Rev. D 69, 086009 (2004), hep-th/0311004; G. Arutyunov, S. Frolov, J. Russo and A. A. Tseytlin, "Spinning strings in $A d S_{5} \times S^{5}$ and integrable systems", Nucl. Phys. B671, 3 (2003), hep-th/0307191.

[26] H. Dimov and R. C. Rashkov, "Generalized pulsating strings", JHEP 0405, 068 (2004), hep-th/0404012.

[27] J. A. Minahan, "Circular semiclassical string solutions on $A d S_{5} \times S^{5}$ ", Nucl. Phys. B648, 203 (2003), hep-th/0209047.

[28] J. Engquist, J. Minahan and K. Zarembo, "Yang-Mills Duals for Semiclassical Strings", JHEP 0311, 063 (2003), hep-th/0310188.

[29] A. Khan and A.L. Larsen, "Spinning pulsating string solitons in $A d S_{5} \times$ $S^{5}$ ", Phys. Rev. D69, 026001 (2004), hep-th/0310019; A. L. Larsen and A. Khan, "Novel explicit multi spin string solitons in $A d S_{5} \times S^{5}$ ", Nucl. Phys. B686, 75 (2004), hep-th/0312184.

[30] M. Smedbäck, "Pulsating strings on $A d S_{5} \times S^{5}$ ", JHEP 0407, 004 (2004), hep-th/0405102.

[31] J. A. Minahan and K. Zarembo, "The Bethe-ansatz for $\mathcal{N}=4$ super Yang-Mills", JHEP 0303, 013 (2003), hep-th/0212208.

[32] G. Arutyunov and S. Frolov, "Integrable Hamiltonian for classical strings on $A d S_{5} \times S^{5 "}$, JHEP 0502, 059 (2005), hep-th/0411089. 
[33] I. Bena, J. Polchinski and R. Roiban, "Hidden symmetries of the $A d S_{5} \times$ $S^{5}$ superstring", Phys. Rev. D 69, 046002 (2004), hep-th/0305116.

[34] N. Beisert, "The dilatation operator of $\mathcal{N}=4$ super Yang-Mills theory and integrability", Phys. Rept. 405, 1 (2005), hep-th/0407277.

[35] M. Kruczenski, "Spin chains and string theory", hep-th/0311203.

[36] M. Kruczenski, A. V. Ryzhov and A. A. Tseytlin, "Large spin limit of $A d S_{5} \times S^{5}$ string theory and low energy expansion of ferromagnetic spin chains", Nucl. Phys. B692, 3 (2004), hep-th/0403120.

[37] R. Hernandez and E. Lopez, "The $S U(3)$ spin chain sigma model and string theory", JHEP 0404, 052 (2004) hep-th/0403139.

[38] R. Hernandez, E. Lopez, A. Perianez and G. Sierra, "Finite size effects in ferromagnetic spin chains and quantum corrections to classical strings", hep-th/0502188; Sakura Schafer-Nameki, Marija Zamaklar, Konstantin Zarembo, "Quantum corrections to spinning strings in $\operatorname{AdS}(5) \times S(5)$ and Bethe ansatz: a comparative study", JHEP 0509 (2005) 051, hepth/0507189; Sakura Schafer-Nameki, Marija Zamaklar, "Stringy sums and corrections to the quantum string Bethe ansatz", JHEP 0510 (2005) 044, hep-th/0509096.

[39] H. Dimov and R.C. Rashkov, "A note on spin chain/string duality", hep-th/0403121.

[40] H. Dimov, V. Filev, R.C.Rashkov, K.S.Viswanathan, "Semiclassical quantization of rotating strings in Pilch-Warner geometry", Phys.Rev. D68 (2003) 066010, hep-th/0304035.

[41] N.P. Bobev, H. Dimov and R.C. Rashkov, "Pulsating strings in warped $A d S_{6} \times S^{4}$ geometry", hep-th/0410262.

[42] R.C.Rashkov and K.S.Viswanathan, "Rotating strings with B-field", hep-th/0211197.

[43] R.C.Rashkov, K.S.Viswanathan and Yi Yang, "Semiclassical analysis of string/gauge duality on non-commutative space", hep-th/0404122. 
[44] S. A. Hartnoll and C. Nunez, "Rotating membranes on G(2) manifolds, logarithmic anomalous dimensions and $\mathcal{N}=1$ duality," JHEP 0302, 049 (2003), hep-th/0210218.

[45] M. Alishahiha and A. E. Mosaffa, "Circular semiclassical string solutions on confining AdS/CFT backgrounds", JHEP 0210, 060 (2002), hepth/0210122;

[46] M. Alishahiha, A. E. Mosaffa and H. Yavartanoo, "Multi-spin string solutions in $A d S$ black hole and confining backgrounds", Nucl. Phys. B686, 53 (2004), hep-th/0402007.

[47] M. Alishahiha and A. Mosaffa, hep-th/0302005.

[48] Hajar Ebrahim and Amir Mosaffa, hep-th/0501072

[49] K. Ideguchi, "Semiclassical strings on $A d S_{5} \times S^{5} / Z(M)$ and operators in orbifold field theories", JHEP 0409, 008 (2004), hep-th/0408014.

[50] F. Bigazzi, A. L. Cotrone and L. Martucci, "Semiclassical spinning strings and confining gauge theories", hep-th/0403261; F. Bigazzi, A.L. Cotrone, L. Matrucci, L.A. Pando Zayas, hep-th/0409205.

[51] D. Aleksandrova and P. Bozhilov, "On the classical string solutions and string /field theory duality", JHEP 0308, 018 (2003), hep-th/0307113; D. Aleksandrova and P. Bozhilov, "On the classical string solutions and string / field theory duality-II", hep-th/0308087.

[52] M. Schvellinger, "Spinning and rotating strings for $\mathcal{N}=1$ SYM theory and brane constructions", JHEP 0402, 066 (2004), hep-th/0309161.

[53] Veselin Filev and Clifford V. Johnson, "Operators with large quantum numbers, spinning strings, and giant gravitons", hep-th/0411023

[54] A. Armoni, J. L. Barbon and A. C. Petkou, "Rotating strings in confining AdS/CFT backgrounds", JHEP 0210, 069 (2002), hep-th/0209224; A. Ramoni, J.L.F. Barbon and A.C. Petkou, JHEP 0206 (2002) 058.

[55] Z. -W. Chong, H. Lu and C. Pope, "Rotating strings in massive type IIA supergravity", hep-th/0402202.

[56] R. P. Andrews, N. Dorey, "Spherical Deconstruction", hep-th/0505107. 